\title{
Orderliness of hormone release patterns: a complementary measure to conventional pulsatile and circadian analyses
}

\author{
Johannes D Veldhuis and Steven M Pincus ${ }^{1}$ \\ Division of Endocrinology, Department of Internal Medicine, Box 202, National Science Foundation Center for Biological Timing, University of \\ Virginia Health Sciences Center, Charlottesville, Virginia 22908, USA and ${ }^{1} 990$ Moose Hill Road, Guilford, Connecticut O6437, USA
}

(Correspondence should be addressed to J D Veldhuis)

\section{Introduction}

Pre-eminent features of physiological hormone release include both pulsatile and nyctohemeral elements, which are modulated in normal physiology and often disrupted in pathological states. The pulsatile nature of neurohormone signaling is critical to long-term activation and physiological regulation of many neuroendocrine axes, such as the gonadotropin-releasing hormone (GnRH)-luteinizing hormone (LH)-gonadal axis in men and women. Indeed, a pulsatile but not continuous GnRH signal to gonadotrope cells will preserve physiological biosynthesis and secretion rates of the gonadotropins, LH and follicle-stimulating hormone (FSH) and, in turn, maintain gonadal steroidogenesis and gametogenesis. For the growth hormone-releasing hormone (GHRH)-somatostatin/growth hormone (GH)/insulinlike growth factor-I (IGF-I) axis, pulsatile GHRH release by hypothalamic neurons is also believed to organize episodic GH secretion, as coordinated by prior and concurrent hypothalamic somatostatin tone. Moreover, the pulsatile $\mathrm{GH}$ signal activates organ-specific secondmessenger signaling pathways, such as the STAT5b signaling cascade in the liver, with subsequent induction of specific genomic responses in relevant target tissues, which are not induced equivalently by a constant GH stimulus. Non-pituitary hormones (e.g. parathyroid hormone (PTH), insulin, renin) also exhibit a distinctly pulsatile time-course of release. Furthermore, neurohormone secretion often partakes of a nyctohemeral and, in some specific cases, circadian rhythmicity. The 24-h rhythms are assembled in part from day-night amplitude and frequency control of the underlying ultradian (shorter-term) pulsations (1). Recent modeling strategies suggest possible mechanisms linking physiological pulsatility and circadian rhythmicity (2), but far more study will be required to establish the bases for such connectivity in health and disease.

\section{Assessing orderliness of hormone release}

In addition to pulsatile and circadian features, another complementary (organizational) aspect of the hormone release process can be quantified. Specifically, the recently developed statistic, approximate entropy (ApEn), quantifies the regularity or orderliness of serial hormone (or other) data, or stated alternatively, the persistence of (sub)pattern features in repetitive measurements over time $(3,4)$. Notably, as applied to endocrine time-series, ApEn will detect alterations in underlying episodic behavior that are not reflected in peak occurrences or amplitudes, a point germane to many applications. Additionally, ApEn provides a direct barometer of feedback change in numerous coupled systems. Many previous studies (both theoretical and clinical) have established good statistical validity (reproducibility) of ApEn for time series of lengths in the order of at least 50 points, even for moderately noisy processes (5-10) and high test-retest reliability (11). This new metric contrasts with so-called deterministic chaos (or nonlinear dynamics), which evaluates in the order of 100000 (or an infinite number of) consecutive noise-free observations and cannot discriminate among short and noisy processes with unequal degrees of subordinate pattern recurrence and/or randomness.

While in multiple contexts pulse identification methods are quite sufficient to characterize and distinguish distinct physiological states, in many other settings there is a need for added analysis beyond monitoring the pulse count and related statistics. For example, pulse identification can be a very challenging endeavor in timeseries of GH secretion in acromegalics (whether or not in partial remission (12)), of $\mathrm{GH}$ in healthy female rats $(10,13)$, of testosterone (especially in older men (6)), of FSH in women (14), and of pathophysiological insulin secretion (8).

In instances where clear pulse identification is difficult, the practical utility of irregularity assessment (e.g. by applying ApEn) is a gain in statistical clarity and replicability of primary inferences. In direct comparison (on common data sets) to algorithms that first require pulse determinations, ApEn analyses in some such circumstances yielded clearer statistical significance between groups, offered similar or greater sensitivity and specificity, and afforded qualitatively new inferences. Descriptively, ApEn thus provides a robust measure of the extent and persistence of distinct levels and types of regularity or orderliness in both primary and subordinate hormone release activity, without 
needing to identify discrete pulses in the process. However, we have found that in other instances, in which pulse identification is relatively clear, ApEn and pulsatility algorithms are largely qualitatively redundant, insofar as extent of capability to distinguish distinct groups. Mechanistically, however, they arise from different perspectives on hormone secretion e.g. individual pulses are taken to reflect underlying discrete secretory events driven by relevant secretagogues and bounded in time, whereas disordered secretory activity (high ApEn values) would suggest loss of coordinated within-system feedback control, increased complexity of regulatory inputs, etc.

We now highlight some representative endocrinological settings illustrating the utility of an irregularity perspective.

\section{LH-FSH dynamics: utility of subordinate level comparisons}

Multiple studies of LH and FSH support the notion of general (albeit imperfect) concordance between the episodic release of these two gonadotropic hormones. Ascertaining LH-FSH secretory differences complementary to and beyond a general 1-1 correspondence between primary LH and FSH pulses may indirectly mark degrees of feedback control, or varying external inputs into the coupled system. We recently found that individual serum FSH concentration time-series are consistently and significantly more irregular than corresponding $\mathrm{LH}$ series in individual young females and young and middle-aged males (14). The capability to quantify this $\mathrm{FSH}-\mathrm{LH}$ difference in irregularity in a statistically valid manner allows one an explicit means to assess both typicality and atypicality (pathophysiology) of the FSH-LH joint dynamics, e.g. across the menstrual cycle, in polycystic ovary syndrome, and with aging. Such assessment can be made either at the secretory level (e.g. after waveform-independent deconvolution analysis, by direct secretion measurements, etc.), or at the more clinically available, yet noisier, serum hormone concentration level. Interestingly, the FSH-LH dichotomy in regularity of release declines progressively with age in men, and is greatly reduced in postmenopausal women (14). Specifically, by ApEn estimates, the difference in the relative irregularity of FSH and LH vanishes postmenopausally. We can thus evaluate the longitudinal evolution of the ApEn(FSH)-ApEn(LH) difference on a per-subject basis, e.g. as a potential marker for the menopausal transition. Men exhibit qualitatively similar results, both in the consistency of young and middle-aged volunteers' greater irregularity of FSH than LH, and in the erosion of this difference in older subjects (14). Therefore, the potential to detect (a)typical joint FSH-LH release dynamics, and to quantify changes in these paired dynamics, via comparisons of FSH and LH irregularity applies to both genders. Indeed, there may be relatively broad utility of this analytical strategy in evaluating aging in males, since mean serum FSH and LH concentrations often do not significantly change with advancing age, thus requiring alternative statistical means of elucidating FSH-LH axis behavior.

\section{Tumoral hormone secretion}

In applications to 24-h serum hormone profiles, ApEn has unveiled distinctions $\left(P<10^{-10}\right.$, nearly $100 \%$ sensitivity and specificity in each study) between healthy controls and tumor-bearing subjects for $\mathrm{GH}$ (12) (normals vs acromegalics), adrenocorticotropin (ACTH) and cortisol (15) (normals vs Cushing's disease), aldosterone (16) (normals vs aldosteronomas), and prolactin (normals vs prolactinomas). In each case, tumoral hormone secretion is markedly more disorderly. This illuminates a general thesis that neuroendocrine tumors are distinguished by reduced regularity of neurohormone release, decreased feedback control, and/or greater strength and/or complexity of (local) regulatory inputs. In addition, ApEn revealed intermediate irregularity of $\mathrm{GH}$ release patterns in patients with treated acromegaly (17). An independent statistical network-based analysis of predictability corroborated this notion of diminished orderliness of $\mathrm{GH}$ release in active acromegaly (18).

The paper by Van den Berg et al. (19) explores the question of persisting disorderliness of $\mathrm{GH}$ secretion in surgically treated acromegalic patients (19). This follow-up study, using an independent GH assay and evaluating a separate patient cohort, corroborates the sentinel insights of Hartman et al. (12), and establishes that enhanced disorderliness of GH release can persist in some patients after clinical and biochemical cure of acromegaly via trans-sphenoidal surgery. Such findings raise the clinically relevant long-term considerations that: (i) the residual normal somatotrope cell mass has recovered incompletely from prior effects of the tumor, (ii) there is a persisting (? nonspecific) impact of neurosurgical intervention, and/or (iii) there are disturbances in the $\mathrm{GH}$ release process imposed by residual tumor cells. Further studies will be required to determine whether (and under what conditions) increased irregularity of $\mathrm{GH}$ release continues for the very long-term postoperatively in acromegalic subjects. In addition, the impact on the orderliness of pituitary hormone secretion of trans-sphenoidal surgical intervention per se will be important to assess. Lastly, the possibility that sustained irregularity of $\mathrm{GH}$ release postoperatively presages later tumor recurrence will require careful longitudinal evaluation. Other tumors will also need to be studied.

\section{Gender differences in orderliness of hormone release}

ApEn-based statistical evaluation of GH secretion patterns in men and women discloses an almost complete 
gender segmentation with women's GH profiles having consistently greater irregularity than men's. ApEn likewise readily discriminates male and female $\mathrm{GH}$ profiles in the adult intact rat (13). More remarkably, in rats that had been castrated prior to puberty, the ApEn of GH profiles in later adulthood is able to separate genetically male and female animals (10). Indeed, among intact animals and rats treated prepubertally either with a longacting GnRH agonist or surgical castration, the following rank order of ApEn of $\mathrm{GH}$ release emerged, listed from maximally disordered to maximally regular: intact female, GnRH-agonist-treated female, ovariectomized female, orchidectomized male, GnRH-agonist-treated male, and intact male (10). Correspondingly, in the human, short-term estrogen and testosterone treatment, but not administration of the non-aromatizable androgen, dihydrotestosterone, stimulated more disorderliness in GH secretion (higher ApEn) in prepubertal girls and boys respectively (9). This response to sex-steroid stimulation emulates that observed (greater disorderliness of GH release) during the later stages of normal puberty in boys $(7,9)$. Such experiments suggest that sex-steroid hormones influence pivotally the interplay within the GHRH-somatostatin/GH/IGF-I feedback-control axis (9). More broadly, quantifying the regularity of neurohormone release by ApEn not only can allow vivid discrimination of normal versus tumoral hormone secretion, but also can uncover and establish graded and oftentimes subtle distinctions.

\section{Aging and disorderly neurohormone release}

Neuroendocrine aging represents a gradual process, the subtle slowly varying features of which have been difficult to delineate quantitatively. Recent applications of ApEn have unmasked consistent and significantly greater secretory disorderliness with advancing age for the $\mathrm{GH}$ axis $(20-22)$, the ACTH axis $(15,23)$, jointly LH and FSH $(5,14)$, individually and jointly $\mathrm{LH}$ and testosterone (6), and insulin release (8). Based on theoretical considerations, the greater disorderliness of hormonal release in healthy aging suggests disruption of within-axis organization or synchrony, and altered feedback control. Indeed, mathematically we can establish that loss of synchrony or coordinate control at the neuronal firing level would often be first manifested qualitatively in an apparent muddying of pulse clarity at the macroscopic level of secretory episodes, i.e. first enhancing subordinate pulse features and nonmonotonic, somewhat 'random' activity, prior to subsequent apparent changes in primary pulse amplitudes and frequencies that only emerge with a substantial further loss of secretory coordination.

Additionally, as a measure of feedback control and coupling strength within a neurohormone axis, the related measure of cross-approximate entropy (crossApEn) has been applied most recently to the aging male hypothalamo-pituitary-gonadal axis, e.g. to evaluate the degree of joint LH-testosterone synchrony (6). CrossApEn quantifies the relative regularity or synchrony (or conditional orderliness) between two hormone release profiles, and does so independently of time lag. The latter feature further distinguishes cross-ApEn from conventional cross-correlation analysis, wherein the time-lag relationship between two hormones must be preserved consistently between the two series in order for significant correlation values to emerge. This distinction can be crucial in biological settings, when the exact timeseparation between coupled neurohormone processes is typically not constant within an individual or across groups of subjects. This theme is highlighted in work by Pincus et al. (6), in which cross-ApEn showed that healthy older men evince significantly asynchronous (uncoupled) LH-testosterone secretion compared with their younger counterparts, while cross-correlation and cross-spectral analyses showed no or less significant younger vs older cohort differences. Similarly, via crossApEn, the ACTH-cortisol axis in healthy men and women shows progressive erosion of bihormonal synchrony with aging (23). Thus cross-ApEn should provide a robust means to assess changes in bihormonal linkage in a large variety of (feedback) networks, including both nonlinear and linear systems.

\section{Future directions and conclusions}

Future directions for understanding the neuroendocrine mechanisms that control orderly hormone release processes include such efforts as developing physiologically based biomathematical models of neuroendocrine feedback axes. Biophysical models should incorporate physiologically appropriate network features, including pertinent types and strengths of feedback and feedforward connections and their interfacing signals, and relevant roles and categories of central control mechanisms (including adaptive control). Ultimate aims are to clarify network topology of physiological axes and routing (pathway) hierarchies, and, more broadly, to implement elements of decision theory and traffic theory (e.g. the possibility of 'broadcast' signaling, priority service, alternative routing), and allow the possibility (and consequences) of 'dropped' or lost messages. Another goal of such physiologically based feedback modeling is to provide a conceptual and analytical basis for eventually monitoring unobserved nodes (or neurohormone control loci) via clinically obtainable information, e.g. predicting GnRH doseresponse curves in vivo given measured hormone profiles for $\mathrm{LH}$ and testosterone (24). Representative issues, among myriad clinical, therapeutic, and diagnostic implications of monitoring hormonal dynamics from an orderliness or regularity vantage, are: (i) how one might restore the orderliness of and feedback within a disrupted axis, (ii) whether early loss of orderliness in hormone secretion will predict tumor recurrence, (iii) 
whether erosion of relative orderliness of LH and FSH release in middle-aged women will presage (preclinically) the onset of the menopause, and (iv) how the quantifiable irregularity of LH release in polycystic ovarian syndrome (25) reflects loss or disruption of GnRH-LH-sex-steroid feedback control mechanisms, etc. These examples are only illustrative and are not meant to be exhaustive.

We conclude that quantitative (statistical) measures of the irregularity or disorderliness of secretory activity within neurohormone axes, e.g. as reflected in the repetitiveness or reproducibility of neurohormone release patterns, can provide novel and insightful assessments that are complementary to evaluating pulsatile (episodic) and 24-h (circadian) variations. We anticipate that further advances in our understanding of neuroendocrine pathophysiology in the human and experimental animals will benefit from these and other strategies for evaluating withinaxis feedback and feedforward control mechanisms, within and between glandular regulation, and bi- and pluri-hormonal synchrony imparted by communicating neurochemical networks that constitute classical endocrine axes.

\section{References}

1 Veldhuis JD, Iranmanesh A, Johnson ML \& Lizarralde G. Twentyfour hour rhythms in plasma concentrations of adenohypophyseal hormones are generated by distinct amplitude and/or frequency modulation of underlying pituitary secretory bursts. Journal of Clinical Endocrinology and Metabolism 1990711616 1623.

2 Keenan D \& Veldhuis JD. A stochastic model of admixed basal and pulsatile hormone secretion as modulated by a deterministic oscillator. American Journal of Physiology: Regulatory, Integrative, and Comparative Physiology 1997273 R1182-R1192.

3 Pincus SM. Approximate entropy as a measure of system complexity (statistic/stochastic processes/chaos/dimension). Proceedings of the National Academy of Sciences of the USA 199188 2297-2301.

4 Pincus SM \& Kalman RE. Not all (possibly) 'random' sequences are created equal. Proceedings of the National Academy of Sciences of the USA 199794 3513-3518.

5 Matt DW, Kauma SW, Pincus SM, Veldhuis JD \& Evans WS. Characteristics of LH secretion in younger versus older premenopausal women. American Journal of Obstetrics and Gynecology 1998 (In Press).

6 Pincus SM, Mulligan T, Iranmanesh A, Gheorghiu S, Godschalk M \& Veldhuis JD. Older males secrete luteinizing hormone and testosterone more irregularly, and jointly more asynchronously, than younger males: dual novel facets. Proceedings of the National Academy of Sciences of the USA 199693 14100-14105.

7 Giustina A, Scalvini T, Tassi C, Desenzani P, Poiesi C, Wehenberg Wet al. Maturation of the regulation of growth hormone secretion in young males with hypogonadotropic hypogonadism pharmacologically exposed to progressive increments in serum testosterone. Journal of Clinical Endocrinology and Metabolism 199782 $1210-1219$.

8 Meneilly GS, Ryan AS, Veldhuis JD \& Elahi D. Increased disorderliness of basal insulin release, attenuated insulin secretory burst mass, and reduced ultradian rhythmicity of insulin secretion in older individuals. Journal of Clinical Endocrinology and Metabolism 199782 4088-4093.
9 Veldhuis JD, Metzger DL, Martha Jr PM, Mauras N, Kerrigan JR, Keenan B et al. Estrogen and testosterone, but not a nonaromatizable androgen, direct network integration of the hypothalamo-somatotrope (growth hormone)-insulin-like growth factor-I axis in the human: evidence from pubertal pathophysiology and sex-steroid hormone replacement. Journal of Clinical Endocrinology and Metabolism 199782 3414-3420.

10 Gevers E, Pincus SM, Robinson ICAF \& Veldhuis JD. Differential orderliness of the $\mathrm{GH}$ release process in castrate male and female rats. American Journal of Physiology 1998 (In Press).

11 Friend KE, Iranmanesh A \& Veldhuis JD. The orderliness of the GH release process and the mass of GH secreted per burst are highly conserved in individual men on successive days. Journal of Clinical Endocrinology and Metabolism 199681 3746-3753.

12 Hartman ML, Pincus SM, Johnson ML, Matthews DH, Faunt LM, Vance ML et al. Enhanced basal and disorderly growth hormone (GH) secretion distinguish acromegalic from normal pulsatile GH release. Journal of Clinical Investigation 199494 1277-1288.

13 Pincus SM, Gevers E, Robinson ICA, Roelfsema F, Hartman ML \& Veldhuis JD. Females secrete growth hormone with more process irregularity than males in both human and rat. American Journal of Physiology 1996270 E107-E115.

14 Pincus SM, Veldhuis JD, Mulligan T, Iranmanesh A \& Evans WS. Effects of age on the irregularity of LH and FSH serum concentrations in women and men. American Journal of Physiology 1997273 E989-E995.

15 Van den Berg G, Pincus SM, Veldhuis JD, Frolich M \& Roelfsema F. Greater disorderliness of adrenocorticotropin and cortisol release accompanies pituitary-dependent Cushing's disease. European Journal of Endocrinology 1997136 394-400.

16 Siragy HM, Vieweg WVR, Pincus SM \& Veldhuis JD. Increased disorderliness and amplified basal and pulsatile aldosterone secretion in patients with primary aldosteronism. Journal of Clinical Endocrinology and Metabolism $19958028-33$.

17 Ovesen P, Vahl N, Fisker S, Veldhuis JD, Orskov H, Christiansen JS et al. Increased growth hormone secretory event frequency and plasma insulin-like growth factor-I levels during the preovulatory interval in normal women: assessment by paired within-subject comparisons, ultrasensitive immunofluorometric GH assay, and deconvolution analysis. Journal of Clinical Endocrinology and Metabolism 1998 (In Press).

18 Prank K, Kloppstech M, Nowlan SJ, Sejnowski TJ \& Brabant G. Self-organized segmentation of time series: separating growth hormone secretion in acromegaly from normal controls. Biophysical Journal 199670 2540-2547.

19 Van den Berg G, Pincus SM, Frolich M, Veldhuis JD \& Roelfsema F. Reduced disorderliness of growth hormone release in biochemically inactive acromegaly after pituitary surgery. European Journal of Endocrinology 1998138 164-169.

20 Veldhuis JD, Liem AY, South S, Weltman A, Weltman J, Clemmons DA et al. Differential impact of age, sex-steroid hormones, and obesity on basal versus pulsatile growth hormone secretion in men as assessed in an ultrasensitive chemiluminescence assay. Journal of Clinical Endocrinology and Metabolism 199580 32093222 .

21 Vahl N, Jorgensen JOL, Skjaerback C, Veldhuis JD, Orskov H \& Christiansen J. Abdominal adiposity rather than age and sex predicts the mass and patterned regularity of growth hormone secretion in mid-life healthy adults. American Journal of Physiology 1997272 E1108-E1116.

22 Friend K, Iranmanesh A, Login IS \& Veldhuis JD. Pyridostigmine treatment selectively amplifies the mass of GH secreted per burst without altering the GH burst frequency, half-life, basal $\mathrm{GH}$ secretion or the orderliness of the GH release process. European Journal of Endocrinology 1997137 377-386.

23 Roelfsema F, Pincus SM \& Veldhuis JD. Patients with Cushing's disease secrete ACTH and cortisol jointly more asynchronously than healthy subjects. Journal of Clinical Endocrinology and Metabolism 1998 (In Press). 
24 Keenan DM \& Veldhuis JD. A biomathematical model of timedelayed feedback in the human male hypothalamic-pituitaryLeydig axis: pulsatile and circadian elements. American Journal of Physiology 1998 (In Press).

25 Rudaz MCG, Ropelato MG, Veldhuis JD, Escobar ME \& Barotini M. Prolonged half life of $\mathrm{LH}$ in adolescents with polycystic ovary syndrome (PCO): assessment by deconvolution analysis. Annual
Meeting of the Latin-American Society of Pediatric Endocrinology, Valdivia, Chile, November 1997 (Abstract).

Received 11 December 1997

Accepted 15 December 1997 